\title{
Vacuum-Assisted Closure: An Effective Technique to Manage Wound Complications After Metastatic Spine Tumour Surgery (MSTS)-A Case Report
}

\author{
RAVISH SHAMMI PATEL, MS (ORTHO), ${ }^{1}$ SAMUEL SHERNG YOUNG WANG, BMED ${ }^{2}$ MIGUEL RAFAEL \\ DAVID RAMOS, MD ${ }^{1}$ HUSAM WALID NAJI NAJJAR, MD ${ }^{3}$ SAMUEL VARA PRASAD, FRCS (ORTHO \& \\ TRAUMA), ${ }^{4}$ NARESH KUMAR, DM (SPINE), FRCS (ORTHO \& TRAUMA) ${ }^{1}$ \\ ${ }^{I}$ Department of Orthopaedic Surgery, National University Hospital, Singapore, ${ }^{2}$ Prince of Wales Clinical School, Faculty of Medicine, University of New South \\ Wales, Sydney, New South Wales, Australia, ${ }^{3}$ Zaqra New Government Hospital, Ministry of Health, Jordan, ${ }^{4}$ Royal National Orthopaedic Hospital, Stanmore, \\ Middlesex, United Kingdom
}

\begin{abstract}
The management of wound complications following metastatic spine tumor surgery (MSTS) remains a formidable task. Plastic coverage procedures after MSTS are challenging due to unhealthy donor sites following previous radiotherapy and prolonged nonambulation. Negative pressure wound therapy (NPWT) is usually not recommended after MSTS due to fear of tumor seeding and excessive blood loss. However, in certain patients post-MSTS, who may be considered as receiving palliative treatment, NPWT can be effective in managing wound complications. We describe our initial experience with the use of NPWT in a 57-year-old lady diagnosed with multiple lumbar and cervicothoracic vertebral metastases secondary to non-small cell lung carcinoma. She underwent 2 cycles of preoperative radiotherapy followed by decompression and posterior instrumentation of lumbosacral and cervicothoracic regions succeeded by another cycle of radiotherapy. The patient developed wound dehiscence and poly-microbial surgical site infection that was not responsive to regular debridements and antibiotics. Hence, we applied NPWT as an alternative treatment to plastic surgical procedures. The patient clinically improved with a reduced quantity of wound discharge, increased granulation tissue, and a downward trend in the inflammatory markers. Subsequently, wound was secondarily closed after 14 days. The patient was discharged after a total hospital stay of 41 days. The intravenous antibiotics (piperacillin/ tazobactam) were changed to oral (ciprofloxacin) after 6 weeks and continued for 4 months. The patient survived for 3 years without any wound complications. Our case report suggests that NPWT can be a potential treatment option for managing wound complications following MSTS.
\end{abstract}

Lumbar Spine

Keywords: spine surgery, metastatis, surgical site infections, wound complications, negative pressure wound therapy, NPWT, vacuum-assisted closure, VAC

\section{INTRODUCTION}

The incidence of postoperative wound infections and complications after metastatic spine tumor surgery (MSTS) have been reported between 1\% and $25 \%{ }^{1}$ Patients undergoing MSTS have hampered wound healing capacity due to previous radiotherapy, chemotherapy, steroids, additional comorbidities, and poor nutrition. ${ }^{2}$ As a result of radical resections, increasing use of implants, alloplastic materials such as polymethylmethacrylate, ceramic spacers, and other bone substitutes, these patients are at high risk of developing debilitating wound complications. ${ }^{3}$ Surgical site infections, repeated debridement, increased perioperative morbidity, prolonged hospital stay, exhaustive health care costs, and mental anguish of patients are the unfortunate results of wound-related issues. ${ }^{4}$ Urgent and definitive plastic surgical procedures such as soft tissue rotational or free flaps with well vascularized tissues are required to protect the exposed implants and the neural structures. ${ }^{5}$ Plastic surgical procedures help to cover the exposed implants, reduce dead space, and increase vascularity followed by improvement in antibiotic delivery and leucocyte function. ${ }^{6}$ However, these plastic surgical procedures are challenging in such patients due to unhealthy donor site secondary to previous radiotherapy and prolonged nonambulation. Owing to the paucity of literature, the optimum strategy to 
manage wound-related complications after MSTS remains unclear.

Negative pressure wound therapy (NPWT) is found effective in managing wound infections, soft tissue loss, vascular insufficiency, and traumatic wounds. ${ }^{7,8}$ It also appears to be safe and effective in the treatment of radiation-associated wound complications. ${ }^{4}$ However, wound complications following exposed malignancy are considered a contraindication for the application of NPWT., 9 In addition, even death has been reported due to excessive blood loss during NPWT after MSTS. ${ }^{11}$ NPWT is usually not recommended after MSTS due to fear of tumor seeding and excessive blood loss. However, in certain patients post-MSTS, who may be considered as receiving palliative treatment, NPWT could still be considered as a possible solution. Successful use of NPWT has been reported after metastatic spinal cord compression or malignant lower limb wounds. ${ }^{10,12}$ The purpose of our case report is to share our initial experience with NPWT in treating wound complication after MSTS.

\section{MATERIALS AND METHODS}

A 57-year-old female without any comorbidities was incidentally noted to have a right upper lobe nodule during health screening. Computed tomography-T-guided biopsy revealed non-small cell lung carcinoma and underwent right upper lobectomy with video-assisted thoracoscopic surgery. She was followed up periodically and was noted to have spinal metastases in the L2-L4 vertebral region a year later. The metastatic lesions were limited to vertebral bodies and did not involve the epidural space. Pedicular biopsy from L3 vertebrae confirmed the diagnosis. Hence, she underwent palliative external beam radiotherapy with 10 sessions of 30 Gy over a period of 15 days in view of vertebral metastases. A year later, she had worsening of her back pain and further investigations revealed an L3 pathological fracture and spread of metastases to $\mathrm{T} 1$ and $\mathrm{S} 1$ with moderate stenosis at the $\mathrm{S} 1$ region. Consequently, she underwent 10 sessions of palliative radiotherapy (30 Gy external beam radiation) over a period of 10 days. Approximately 2 months after radiotherapy, she developed sudden onset left lower limb weakness, urinary retention, and constipation. Magnetic resonance imaging revealed multiple vertebral metastases in C6, T1, T11-L1, L3, and $\mathrm{S} 1$ with concomitant pathological fractures with cord compression at C6 and L1. She underwent posterior instrumentation from $\mathrm{C} 3$ to $\mathrm{T} 3$ and decompression from C6 to T1. Postoperatively, she received dexamethasone for 24 hours and prophylactic antibiotics (cefazolin) were continued for 48 hours. She underwent preoperative embolization for metastases in L1 to L3 followed by posterior decompression at L1 and L3 and posterior instrumentation from $\mathrm{T} 9$ to ilium 3 days later (Figure 1). She suffered from urinary tract infection on the ninth postoperative day and was treated with imipenem and ciprofloxacin for Enterococcus faecalis and Morganella. She was discharged on the 16th postoperative day without any wound issues. Gefitinib was started, and she was planned for 10 sessions of palliative radiotherapy to lumbosacral spine from 21st postoperative day onwards.

After completion of 7 cycles of radiotherapy, lumbosacral wound dehiscence with a serous discharge was observed and magnetic resonance imaging findings were consistent with early infection. The patient underwent 3 wound washes and debridements over a period of 15 days. Polymicrobial infection with Klebsiella pneumoniae, Pseudomonas aeruginosa, Staphylococcus aureus, and Acinetobacter baumannii were identified on culture assays. The patient was treated with longterm intravenous piperacillin/tazobactam and gentamicin $(160 \mathrm{mg}$ in $20 \mathrm{~mL}$ ) was applied to the wound as per the antibiotic sensitivity report. However, there was no improvement in the wound condition, and the inflammatory markers remained persistently elevated. During the fourth debridement, (Figure 2) V.A.C. (Kinetic Concepts, Inc, San Antonio, Texas) was applied over VersaFoam white sponge (Kinetic Concepts, Inc; Figure 3a).

\section{V.A.C. Application Procedure}

This was carried out in the operating theatre under general anesthesia, with aseptic precautions. The patient was positioned prone on a Jackson Table. The previous surgical scar was opened and wound debrided. The wound was washed out with $2 \mathrm{~L}$ of warm saline (Figure 2). The wound was partially closed with a lower portion left open for the application of NPWT. Polyurethane sponge foam (VersaFoam) was cut to $10 \times 5 \mathrm{~cm}$ and placed on the left side; while a smaller sized $7.5 \times 2.5 \mathrm{~cm}$ was placed on the right side of the spinous process, in the distal end of the incision. A last strip of VersaFoam was applied horizontally across the exposed dura at the L3 laminectomy site for 


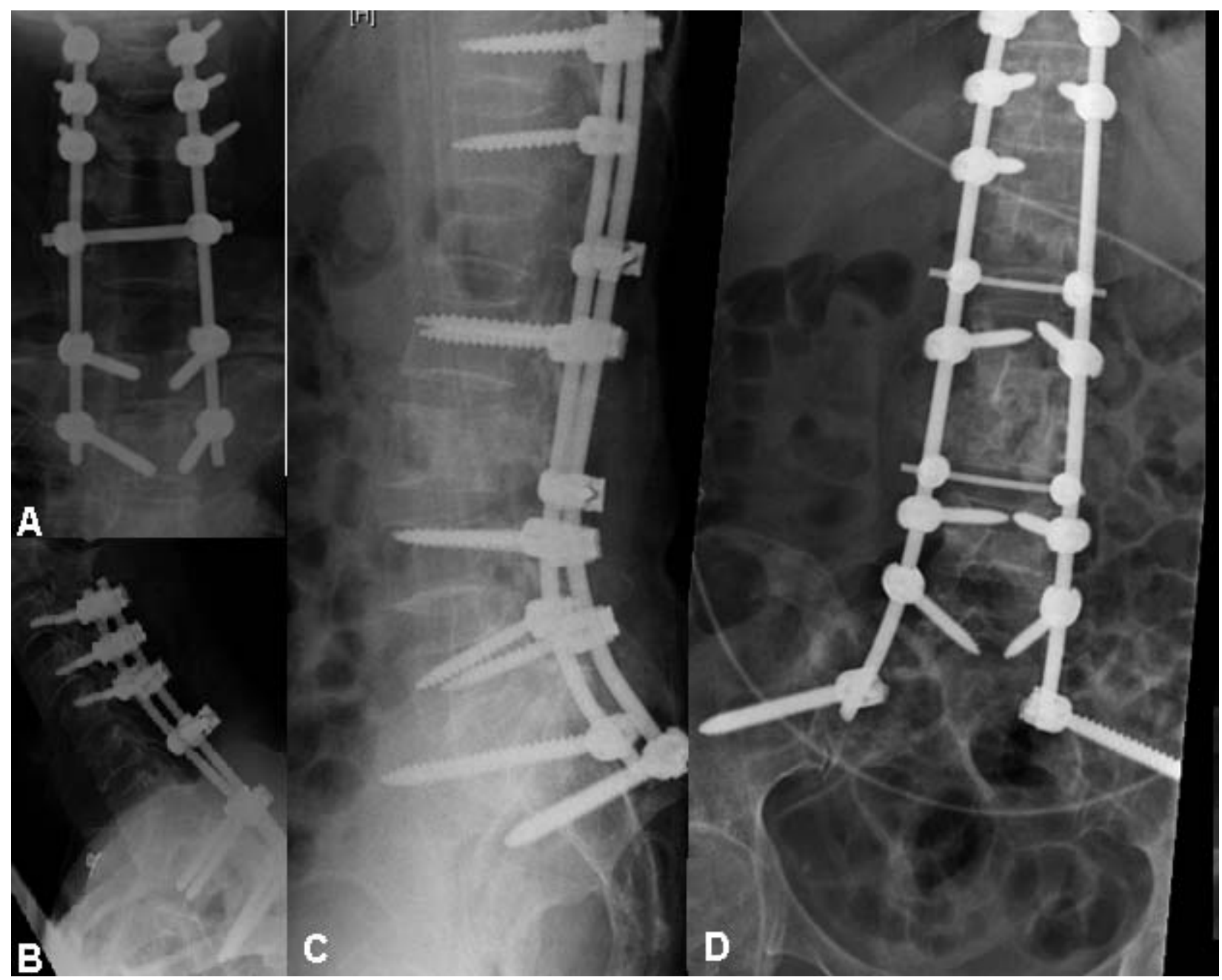

Figure 1. Postoperative radiograph after posterior instrumentation from T9 to ilium.

protection from the unwanted effects of negative pressure at this delicate area. Figures $4 \mathrm{a}$ and $4 \mathrm{~b}$ provide a visual representation of the arrangement of the VersaFoam white sponge in the wound. Final black V.A.C sponge was placed on the incision vertically and over the previous drain site horizontally. The area was sealed with large strips of air

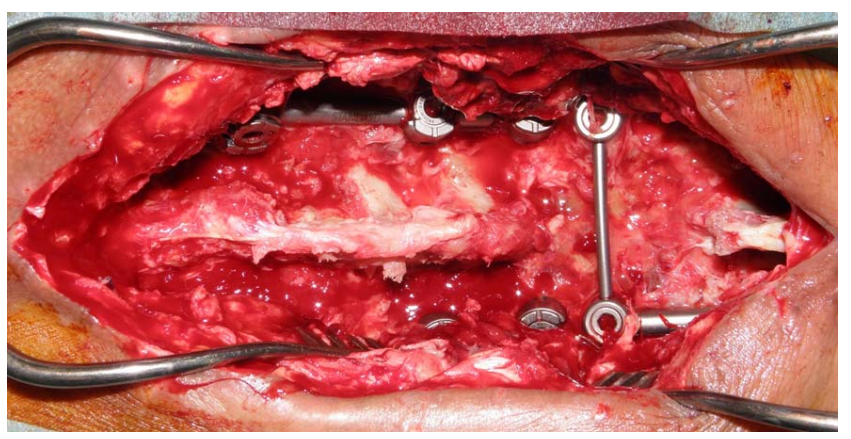

Figure 2. Postoperative wound after fourth debridement and before application of V.A.C. tight dressing. A connecting tube surrounded by another plastic sealant was attached to a cut open area of the previous plastic sealant and was subjected to a subatmospheric pressure of -125 $\mathrm{mm} \mathrm{Hg}$ continuously. A pressure bandage was applied over the wound in order to facilitate the negative pressure.

The patient was nursed in a lateral decubitus or prone position while on NPWT. The sponge was changed on a twice weekly basis. The patient was monitored twice daily for the amount of blood loss, and every alternate day inflammatory markers were repeated to obtain the trend. Wound swabs were taken every time the V.A.C. dressing was changed, and the dressing was continued until the formation of healthy granulation tissue; down trending inflammatory markers and wound cultures did not grow any organism. Serous discharge reduced significantly, and granulation tissue increased over a period of 2 weeks. The inflammatory markers 


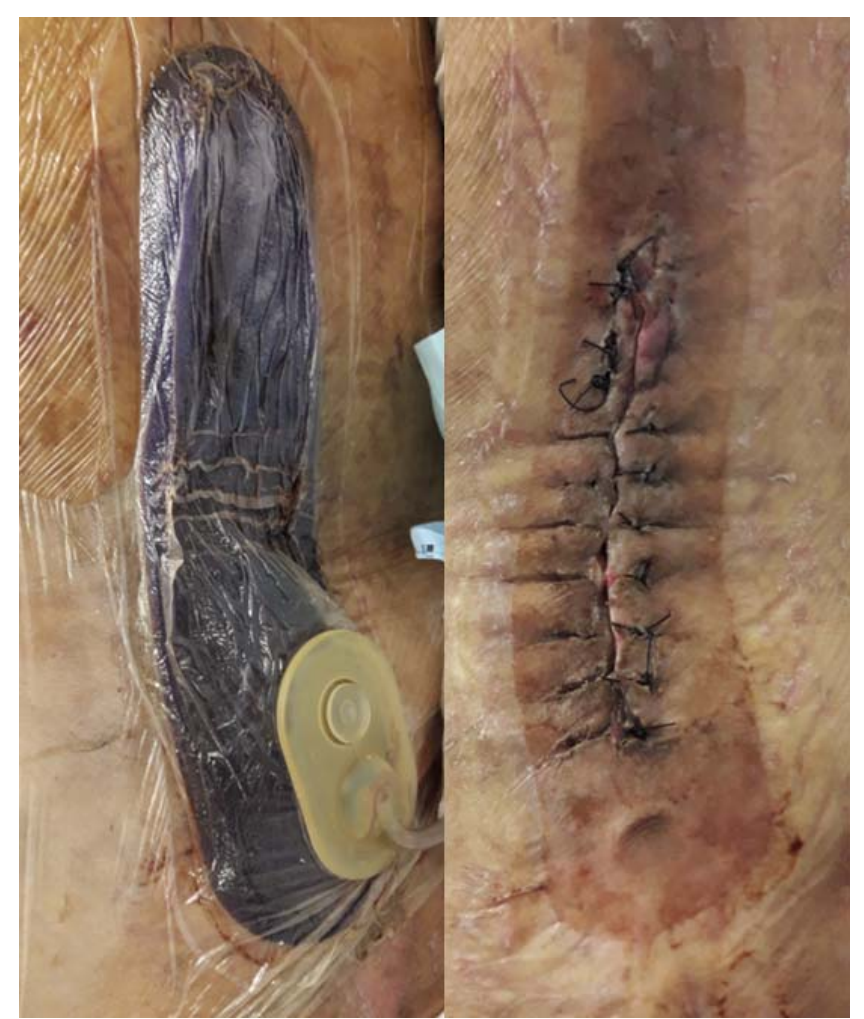

Figure 3. ( $a, b)$ Left side picture showing application of V.A.C.; right side picture showing wound closure with nonabsorbable sutures after 2 weeks of V.A.C. therapy.

showed a downward trend, and the hemoglobin and total protein improved significantly. After 14 days of NPWT, the wound was secondarily closed, and sutures were removed after 15 days (Figure $3 \mathrm{~b}$ ).

Upon discharge, total hospital stay was 41 days. Dual oral antibiotic therapy was prescribed for another 6 weeks while only ciprofloxacin was continued for a period of 4 months as per the infectious disease specialists. She was followed up for a period of 3 years without any wound complications nor clinical and radiological evidence of tumor seeding at the postoperative site. She developed a pathological fracture of left hip that was treated with total hip replacement, also noted to have further metastases in the cervical spine with kyphotic deformity, but she refused further intervention. She died 3 years after the MSTS due to type I respiratory failure secondary to pulmonary embolism with background of metastatic non-small cell lung carcinoma.

\section{DISCUSSION}

NPWT is an effective adjuvant treatment in treating surgical site infections and wound dehiscence. ${ }^{10}$ Several factors are responsible for the

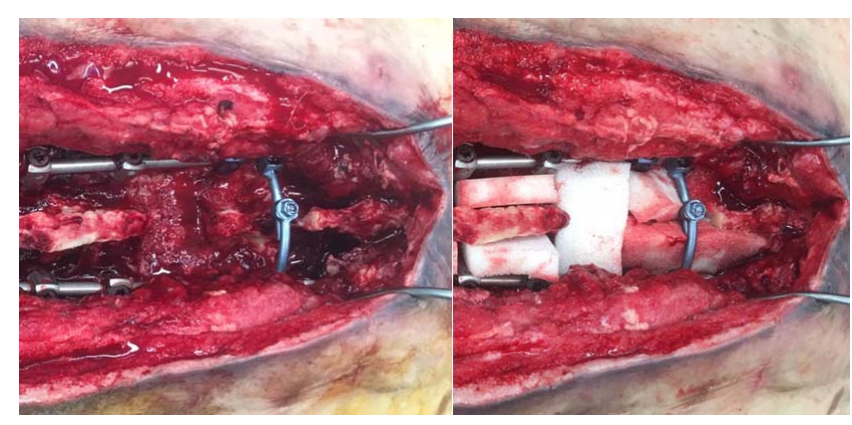

Figure 4. (a, b) Representation of VersaFoam white sponge arranged over dura and along spinous processes.

effectiveness of negative pressure dressing, but the exact mechanism remains unclear. ${ }^{11}$ NPWT therapy is based on the work of Dersch et $\mathrm{al}^{13}$ concluding that positive pressure reduces skin oxygen tension and the negative pressure increases perfusion. Subsequently, animal studies were carried out to evaluate the utility of NPWT in infection control and wound healing. Morykwas et $\mathrm{al}^{8}$ further studied the effect of NPWT on wounds in pig model and found that NPWT optimizes the blood flow to the wound, reduces the bacterial load, and facilitates removal of interstitial fluid, thereby promoting granulation. Consistent with the animal studies, similar effects have been observed during in vivo human applications. In a clinical setting, NPWT reduces the surface area of the wound, facilitates debridement of necrotic tissues, promotes bacterial clearance, removes edema fluid, acts as a sterile barrier that prevents further contamination, and promotes neovascularization. ${ }^{7,14,15}$ As a combined effect of all the mechanisms, there is rapid formation of abundant granulation tissue. .,10,14 $^{2}$

Numerous retrospective series and case reports have highlighted the effectiveness of NPWT for the management of wound complications after spinal instrumentation. ${ }^{10}$ However, several authors and manufacturers have considered the presence of malignancy to be a contraindication for the use of NPWT. ${ }^{5,10,16}$ NPWT has potential to promote tumor growth and has theoretical risk of accelerating neoplastic spread and tumor seeding. ${ }^{10}$ The caution exhibited by these previous studies is based more on hypothetical fear rather than actual evidence of metastatic spread to muscle and subcutaneous tissue during the NPWT process. In fact, we postulate that NPWT decreases the local tumor burden via the same mechanism by which bacterial load and likelihood of colonization are reduced $^{8,10}$ by drawing malignant cells in the third 
space fluid directly into the NPWT apparatus. Regular clinical and radiological surveillance of our patient's wound yielded negative results for the additional 3 years of survival. Jones et $\mathrm{al}^{11}$ in a retrospective case series, reported a death associated with the use of NPWT for postoperative wound infection following MSTS. The patient had intraoperative blood loss that continued in the postoperative period during NPWT application. After 2 days of NPWT, the patient became hemodynamically unstable and could not be resuscitated as he refused blood transfusion citing religious reasons. The primary reason in this case may have been the inability to revive the patient without blood transfusion. Not keeping with the above finding, successful use of NPWT has been reported by Adams et $\mathrm{al}^{17}$ for the treatment of wound complications in patients with malignant desmoplastic melanoma and neurofibromatosis. The use of NPWT in a palliative situation has been reported by Ford-Dunn ${ }^{18}$ and Riot et al. ${ }^{12}$ NPWT was used to control exudates and to reduce pain during palliative treatment of malignant wounds. ${ }^{19}$

Theoretically, NPWT is believed to potentially cause adverse effects like on-going pain due to sponge volume and negative pressure, hypertrophy of granulation tissue over the sponge, damage to important adjacent vessels, minor bleeding at dressing changes, odor, and skin erosion around the suction tube. ${ }^{20-24}$ However, we did not encounter any such complications in our case. This has also been reiterated by Mermerkaya et $\mathrm{al}^{25}$ and Karaaslan et $\mathrm{al}^{26}$ who did not observe the above mentioned complications. NPWT is strictly contraindicated in the presence of active cerebrospinal fluid leak, bleeding diathesis, and in patients with an allergy to the NPWT dressing; and relatively contraindicated in metastatic or neoplastic disease in the wound. ${ }^{10}$ However, in patients undergoing palliative treatment, the use of NPWT for managing wound complications is justified. ${ }^{12,18}$ Such patients need to be frequently monitored for excessive blood loss, cerebrospinal fluid leak, neurodeficit, and sepsis. NPWT can be a potential alternative to complex plastic surgical procedures or conventional wound management, thereby enabling faster wound healing, reducing number of debridements, shortening hospital stay, reducing health care cost, and improving quality of life of the patients in their terminal stage.
Similar to wound complications that are seen in patients with terminal illnesses, patients undergoing MSTS are also predisposed to a higher incidence of wound infection and wound dehiscence. ${ }^{27}$ Our case highlights a typical scenario of the same, in a patient who had undergone radiotherapy to the lumbar spine, followed by MSTS, to address the persistent disease. The patients undergoing radiation before MSTS may carry a risk of wound-related complications of up to $32 \% .^{27,28}$ This risk is because radiotherapy affects the healing potential of the skin and predisposes it to an array of wound complications that are difficult to manage. ${ }^{28}$ Mustoe and Porras-Reyes $^{29}$ have experimentally proven that radiation inhibits fibroblasts, where the latter forms an essential component for the formation of collagen in surgical wounds. Both preoperative and postoperative radiation therapy can lead to wound complications, but a higher rate has been observed with a preoperative radiotherapy (35\%) than postoperative radiotherapy $(17 \%) .{ }^{30}$ Thus, patients undergoing perioperative radiotherapy have high risk of wound complications, and plastic surgical procedures can be difficult and challenging. NPWT has a potential to be effective in managing wound complications of these patients.

We envisage that use of NPWT in postoperative wound dehiscence for MSTS, wherever feasible, will reduce the burden of surgical morbidity, number of operation theatre visits, length of hospital stay, and overall treatment cost. Timing of application of NPWT is ultimately surgeon dependent, but knowledge of factors contributing to MSTS wound complications can theoretically make it a viable treatment option at the first or second wound debridement. Our patient underwent 5 visits to the operation theatre, as we decided to use NPWT only in her fourth visit. Had this decision been made earlier, the number of operative visits might have been brought down to 2 or 3 , from 5. This is supported by Mehbod et $\mathrm{al}^{31}{ }^{31}$ who showed that application of NPWT assisted closure reduced the number of operative visits to 2.2 from 4.7 visits for patients undergoing standard wound debridement and closure. Our case also highlights that application of V.A.C. dressing has to be modified so that the suction of the V.A.C. system is transmitted over 2 sets of VersaFoam white sponge and is not directly over the dura or bleeding surface. This modification in application of V.A.C. dressing makes it safer to be used in MSTS. 


\section{CONCLUSION}

We recommend that there is a place for NPWT in management of postoperative wound complications after MSTS. Regardless of oncological staging and prognosis, if patients are deemed medically fit to undergo a major oncological surgical procedure, we do not see any contraindication in the use of NPWT as salvage for wound dehiscence or failure. Quality of life in patients with closed surgical wounds is assuredly better than ones that require multiple interventions and trips to the operating theatre. ${ }^{32}$ We strongly agree with Ousey et $\mathrm{al}^{10}$ that further studies are needed to demonstrate the economic feasibility/cost-benefit ratio of using NPWT in treating postoperative wound complications.

\section{ACKNOWLEDGMENT}

We acknowledge Dr Nivetha Kumar for her contribution to this article.

\section{REFERENCES}

1. Mesfin A, Sciubba DM, Dea N, et al. Changing the adverse event profile in metastatic spine surgery: an evidencebased approach to target wound complications and instrumentation failure. Spine (Phila Pa 1976). 2016;41(suppl 20):S262S70.

2. Itshayek E, Yamada J, Bilsky M, et al. Timing of surgery and radiotherapy in the management of metastatic spine disease: a systematic review. Int J Oncol. 2010;36(3):533-544.

3. Chang DW, Friel MT, Youssef AA. Reconstructive strategies in soft tissue reconstruction after resection of spinal neoplasms. Spine (Phila Pa 1976). 2007;32(10):1101-1106.

4. Siegel HJ, Long JL, Watson KM, Fiveash JB. Vacuumassisted closure for radiation-associated wound complications. J Surg Oncol. 2007;96(7)575-582.

5. Yuan-Innes MJ, Temple CL, Lacey MS. Vacuum-assisted wound closure: a new approach to spinal wounds with exposed hardware. Spine (Phila Pa 1976). 2001;26(3):E30-E33.

6. Calderon W, Chang N, Mathes SJ. Comparison of the effect of bacterial inoculation in musculocutaneous and fasciocutaneous flaps. Plast Reconstr Surg. 1986;77(5):785-794.

7. Venturi ML, Attinger CE, Mesbahi AN, Hess CL, Graw KS. Mechanisms and clinical applications of the vacuumassisted closure (VAC) device. Am J Clin Dermatolo. 2005;6(3):185-194.

8. Morykwas MJ, Argenta LC, Shelton-Brown EI, McGuirt W. Vacuum-assisted closure: a new method for wound control and treatment: animal studies and basic foundation. Ann Plast Surg. 1997;38(6):553-562.

9. Argenta LC, Morykwas MJ. Vacuum-assisted closure: a new method for wound control and treatment: clinical experience. Ann Plast Surg. 1997;38(6):563-576; discussion 77.

10. Ousey KJ, Atkinson RA, Williamson JB, Lui S. Negative pressure wound therapy (NPWT) for spinal wounds: a systematic review. Spine J. 2013;13(10):1393-1405.

11. Jones GA, Butler J, Lieberman I, Schlenk R. Negativepressure wound therapy in the treatment of complex postoperative spinal wound infections: complications and lessons learned using vacuum-assisted closure. J Neurosurg Spine. 2007;6(5):407-411.

12. Riot S, de Bonnecaze G, Garrido I, Ferron G, Grolleau J-L, Chaput B. Is the use of negative pressure wound therapy for a malignant wound legitimate in a palliative context? "The concept of NPWT ad vitam": a case series. Palliative Med. 2015;29(5):470-473.

13. Dersch T, Morykwas M, Clark M, Argenta L, eds. Effects of negative and positive pressure on skin oxygen tension and perfusion. 4th Annual Meeting of Wound Healing Society. San Francisco, CA; 1994.

14. Saxena V, Hwang CW, Huang S, Eichbaum Q, Ingber D, Orgill DP. Vacuum-assisted closure: microdeformations of wounds and cell proliferation. Plast Reconstr Surg. 2004;114(5):1086-1096; discussion 97-98.

15. Weed T, Ratliff C, Drake DB. Quantifying bacterial bioburden during negative pressure wound therapy: does the wound VAC enhance bacterial clearance? Ann Plast Surg. 2004;52(3):276-279; discussion 9-80.

16. Place HM, Zehnder SW. Vacuum-assisted wound closure in postoperative spinal wound infection. Orthopedics. 2007;30:4.

17. Adams J, Hakim E, Hakim D, Myers B, Horowitz J. A critical analysis of existing evidence on the management of a wound with malignant desmoplastic melanoma and neurofibromatosis: a case report. Rehab Oncol. 2009;27(1):26-27.

18. Ford-Dunn $\mathrm{S}$. Use of vacuum assisted closure therapy in the palliation of a malignant wound. Palliative Med. 2006;20(4):477-478.

19. Ploumis A, Mehbod AA, Dressel TD, Dykes DC, Transfeldt EE, Lonstein JE. Therapy of spinal wound infections using vacuum-assisted wound closure: risk factors leading to resistance to treatment. Clin Spine Surg. 2008;21(5):320-323.

20. DeFranzo A, Argenta L, Marks M, et al. The use of vacuum-assisted closure therapy for the treatment of lowerextremity wounds with exposed bone. Plast Reconstr Surg. 2001;108(5):1184-1191.

21. Argenta LC, Morykwas MJ. Vacuum-assisted closure: a new method for wound control and treatment: clinical experience. Ann Plast Surg. 1997;38(6):563-577.

22. Masden D, Goldstein J, Endara M, Xu K, Steinberg J, Attinger C. Negative pressure wound therapy for at-risk surgical closures in patients with multiple comorbidities: a prospective randomized controlled study. Ann Surg. 2012;255(6):1043-1047.

23. Senchenkov A, Petty PM, Knoetgen J, Moran SL, Johnson CH, Clay RP. Outcomes of skin graft reconstructions with the use of Vacuum Assisted Closure (VAC $\left.{ }^{\circledR}\right)$ dressing for irradiated extremity sarcoma defects. World J Surg Oncol. 2007;5(1):138.

24. Gopal S, Majumder S, Batchelor A, Knight S, De Boer P, Smith R. Fix and flap: the radical orthopaedic and plastic treatment of severe open fractures of the tibia. Bone Joint J. 2000;82(7):959-966.

25. Mermerkaya U, Bekmez S, Alkan E, Ayvaz M, Tokgozoglu M. Evaluation of vacuum-assisted closure in 
patients with wound complications following tumour surgery. Int Wound J. 2016;13(3):394-397.

26. Karaaslan F, Erdem Ş, Mermerkaya MU. Wound management with vacuum-assisted closure in postoperative infections after surgery for spinal stenosis. Int Med Case Rep J. 2015;8:7-11.

27. Ghogawala Z, Mansfield FL, Borges LF. Spinal radiation before surgical decompression adversely affects outcomes of surgery for symptomatic metastatic spinal cord compression. Spine. 2001;26(7):818-824.

28. Wang J, Boerma M, Fu Q, Hauer-Jensen M. Radiation responses in skin and connective tissues: effect on wound healing and surgical outcome. Hernia. 2006;10(6):502-506.

29. Mustoe T, Porras-Reyes B. Modulation of wound healing response in chronic irradiated tissues. Clin Plast Surg. 1993;20(3):465-472.

30. O'Sullivan B, Davis AM, Turcotte R, et al. Preoperative versus postoperative radiotherapy in soft-tissue sarcoma of the limbs: a randomised trial. Lancet. 2002;359(9325):2235-2241.

31. Mehbod A, Ogilvie J, Pinto M, et al. Postoperative deep wound infections in adults after spinal fusion: management with vacuum-assisted wound closure. Clin Spine Surg. 2005;18(1):1417.

32. Kumar N, Malhotra R, Zaw AS, et al. Evolution in treatment strategy for metastatic spine disease: presenting evolving modalities. Eur J Oncol. 2017;43(9):1784-1801.

Disclosures and COI: The authors received no funding for this study and report no conflicts of interest.

Corresponding Author: Associate Professor Naresh Kumar, Department of Orthopaedic Surgery, University Orthopaedics, Hand \& Reconstructive Microsurgery Cluster, 1E Kent Ridge Rd, NUHS Tower Block, Level 11, Singapore 119228. Phone: (65) 6-772-5611;Fax: (65) 6-778-0720;Email: dosksn@nus.edu.sg.

Published 31 December 2019

This manuscript is generously published free of charge by ISASS, the International Society for the Advancement of Spine Surgery. Copyright (C) 2019 ISASS. To see more or order reprints or permissions, see http://ijssurgery.com. 